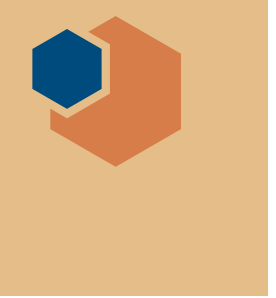

\title{
US DOE clean energy initiative designed to accelerate materials to market
}

\author{
http://energy.gov/eere/energy-materials-network
}

\begin{abstract}
A dvanced materials solutions have the potential to revolutionize the energy landscape and tip the scales further toward clean energy technologies. One of the most significant hurdles in the advancement of clean energy lies in bringing innovative new products to market, a process that often takes longer than a decade. The traditionally slow pace to discover, develop, test, and commercialize advanced materials solutions for clean energy will leave the United States falling short of its ambitious goals for carbon reduction and runs counter to efforts to reverse the decline of US manufacturing. In an attempt to accelerate the materials development cycle, the US Department of Energy (DOE) launched the Energy Materials Network (EMN) in February 2016.

The Energy Materials Network is part of a broader effort by the Obama administration to develop a clean energy
\end{abstract}

economy and to reestablish a vibrant manufacturing sector in the United States through advanced manufacturing. Both the Materials Genome Initiative (to discover and deploy advanced materials twice as fast at a fraction of the cost) and the recommendations of the Advanced Manufacturing Partnership 2.0 (which highlights the importance of materials development for US-based advanced manufacturing) are supported by this new network program

A national laboratory-led initiative, the EMN partners the laboratories with industry to tackle some of the most pressing materials-related challenges for clean energy manufacturing. Four initial critical areas of materials development have been identified, and $\$ 40$ million in funding for fiscal year 2016 has been provided by the DOE's Office of Energy Efficiency and Renewable Energy (EERE) to establish the first four consortia.
The Lightweight Materials Consortium (LightMAT) is led by Pacific Northwest National Laboratory (PNNL) and focuses on the development and application of lightweight materials to increase fuel efficiency for vehicles. Jointly led by Argonne National Laboratory and Los Alamos National Laboratory (LANL), the Electrocatalysis Consortium (ElectroCat) seeks to replace the rare and expensive platinum group metals in fuel cells with next-generation electrocatalysts. The Caloric Cooling Consortium (CaloriCool) is led by Ames Laboratory and is working to discover new caloric materials (materials that cool when subjected to magnetic, electric, or mechanical forces) and to develop more energy-efficient cooling technologies.

A fourth consortium to develop cheaper and more durable materials for solar modules will be established later this year. An additional $\$ 120$ million has also been requested for fiscal year 2017 to further support the original four consortia and to add an addi-

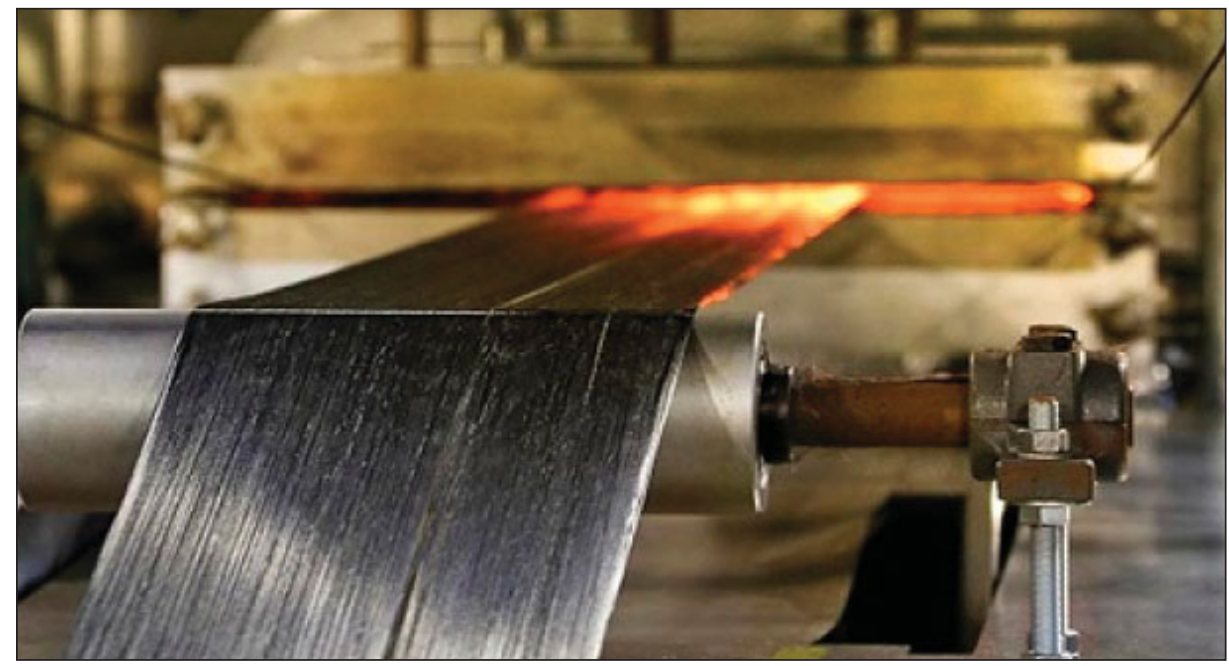

Established as part of the Energy Materials Network, the mission of the Lightweight Materials National Laboratory Consortium (LightMAT) is to create an enduring national lab-based network, enabling industry to utilize the national laboratories' unique capabilities related to lightweight materials. Photo credit: Oak Ridge National Laboratory. tional three that will focus on catalysts for biofuels and bioproducts, materials for renewable hydrogen production, and materials for lowpressure hydrogen storage.

The consortia-based structure of the EMN establishes focus around key materials-related clean energy manufacturing challenges and organizes the research and development capabilities, facilities, and expertise at the national laboratories around these issues. Each consortium acts as a single point of contact for a specific technological challenge, making federal resources more readily available to industry partners. This approach is meant to yield a significantly shorter and more 
cost-effective route from the laboratory to the market by facilitating access and by incorporating industry-specific issues (such as manufacturing and scale-up) into the materials development process.

According to Iver Anderson, Senior Metallurgist at Ames Laboratory and a member of the LightMAT steering committee, "There are many scientists within the national labs who are eager to work on real applications for their materials discoveries and processing inventionsto reach the ultimate technology goal line." Anderson also points out the frustrations experienced on the industry side with engineers and technology managers often knowing about, but having difficulty accessing, the technology and expertise within the national laboratory network. "Closing this information gap and rapidly enabling effective partnerships to provide a clear path to solve industry's critical problems is the EMN mission," Anderson says.

"The EMN is a tremendous move by the DOE at removing barriers - perceived and actual - from industry to accelerate work with the national laboratories," says Yuri Hovanski, Director of LightMAT at PNNL. Hovanski characterized the access the EMN provides for industry to the national laboratories as "strategic and unprecedented" and explained how the EMN is changing the game with a realworld materials example:

Before the EMN, if a company needed to better understand new powder streams for additive manufacturing being developed at Ames, they would have to access high speed computing from ORNL [Oak Ridge National Laboratory], predictive modeling from LANL, and simulation validation from PNNL - all with separate contracts and unique agreements. This often hindered industry from accessing the very best team possible at overcoming their challenges. Since the advent of the EMN, a single agreement with LightMAT allows companies to access all these capabilities - and many more - seamlessly across the ten partnering labs in our consortium.

While the recent establishment of the EMN means the consortia are in vari- ous stages of readiness, LightMAT and ElectroCat have both launched websites that list the capabilities housed within the network as well as relevant access details. W. Jud Ready, Deputy Director of the Institute for Materials at the Georgia Institute of Technology, works on electronic applications of nanomaterials, and noted that while he has not yet had a chance to collaborate with the EMN in his research, "one of the most useful items has simply been the cataloguing of available equipment and facilities and how to access them with an actual name, phone, email, and webpage associated with it."

Ready expects the EMN to have a positive impact on the materials community through increased "opportunities for funding, collaboration, and equipment use." And according to Hovanski, the potential for the EMN to integrate and accelerate the materials development cycle would "enable US manufacturers to deliver innovative, made-in-America products to the world market."

Jennifer A. Nekuda Malik
European cities test green energy and transport solutions www.remourban.eu

$\mathrm{T}$ he project Regeneration Model for Accelerating the Smart Urban Transformation (REMOURBAN), funded by the European Union (EU), will demonstrate strategies that European cities can use to reduce energy consumption, improve transport sustainability, and cut greenhouse gas emissions. The aim is to improve the quality of life in urban areas through cooperative efforts on green energy and transport solutions.

The project's approach encourages individuals and communities across Europe to work together on implementing sustainable, green solutions to meet energy and transport needs. The approach is in line with the EU's plan to develop smart cities that integrate information and communication technologies (ICTs) with sustainable energy and transport solutions.
REMOURBAN is implementing and testing a range of green energy and transport strategies in three partner cities: Valladolid (Spain), Nottingham (UK), and Tepebaşi/Eskişehir (Turkey). The project will work closely with city administrations and residents to develop these strategies, based on local needs in selected districts.

The project is targeting energy savings of $50-53 \%$ and emission reductions of 26-80\%, involving between 5700 and 8100 residents depending on the participating city district. The project's work includes:

- retrofitting buildings and improving lighting and equipment to achieve energy savings;

- installing domestic and district-wide heating systems that use renewable sources integrated with smart energy monitoring and control technologies;

- deploying electric and hybrid vehicles and buses in place of conventional fuel-powered ones, along with associated charging stations and management technologies; and

- developing information and communication technologies and platforms to manage city infrastructure, smart energy grids, and traffic.

The approaches will be shared across the three test cities, and with two other project partners-Seraing (Belgium) and Miskolc (Hungary) - as a means of developing generic solutions that can be applied elsewhere across Europe.

REMOURBAN will also identify the main barriers to achieving urban sustainability goals and propose regulation adjustments that will encourage cities to switch to green energy and transport solutions, and to improve public procurement procedures. 